\title{
ANÁLISE DOS INDICADORES DE HEMORRAGIA LETAL EM VÍTIMAS DE TRAUMA PENETRANTE DE TRONCO ADMITIDAS EM CHOQUE: UM MÉTODO OBJETIVO PARA SELECIONAR OS CANDIDATOS AO "CONTROLE DE DANOS"
}

\author{
ASSESSING THE RISK OF DEATH DUE TO HEMORRHAGE IN VICTIMS OF \\ PENETRATING TRAUMA TO THE TORSO ADMITTED IN SHOCK: AN OBJECTIVE \\ METHOD TO SELECT CANDIDATES FOR DAMAGE CONTROL
}

\author{
José Gustavo Parreira, TCBC-SP1 \\ Sílvia Cristine Soldá, TCBC-SP² \\ Samir Rasslan, TCBC-SP 3
}

\begin{abstract}
RESUMO: Objetivo: Identificar os indicadores de hemorragia letal em vítimas de trauma penetrante de tronco, admitidas com hipotensão arterial sistêmica e analisar sua aplicabilidade na seleção dos candidatos ao "controle de danos". Método: Foram analisadas informações sobre 74 vítimas de ferimentos penetrantes exclusivamente de tronco, admitidas com hipotensão arterial sistêmica secundária à hemorragia, que sobreviveram até o tratamento definitivo. Os dados foram coletados prospectivamente durante dois anos. A média etária foi $29,5 \pm 8$ anos, e $62(83 \%)$ pacientes eram do sexo masculino. Trinta e nove (52\%) foram vítimas de ferimentos de instrumentos perfurocortantes e 35 (47\%), de ferimentos por projéteis de arma de fogo. Houve 23 óbitos $(31 \%), 19$ por hemorragia $(82,6 \%)$. Os que faleceram por hemorragia foram incluídos no grupo $\mathrm{H}$ e os outros no grupo O. Foram comparadas diversas variáveis entre os grupos, utilizando-se o teste $t$ de Student (controlado pelo teste de Levene) e a correlação de Spearman, considerando $\mathrm{p}<0,05$ como significativo. Resultados: As variáveis de maior correlação com hemorragia letal foram a pressão arterial sistólica no início da operação $(<110 \mathrm{mmHg})$, o pH arterial no início da operação $(<7,25)$, a resposta à infusão endovenosa de líquidos à admissão (choque persistente) e o volume de concentrados de hemácias transfundido durante a operação $(>1.200 \mathrm{ml})$. Através de um modelo de regressão logística foi possível calcular o risco de morte por hemorragia baseado na pressão arterial no início da operação e volume de concentrados de hemácias transfundido. Conclusão: A análise dos indicadores de hemorragia letal fornece dados objetivos para a indicação do "controle de danos".
\end{abstract}

Descritores: Ferimentos penetrantes; Choque; Choque hemorrágico; Mortalidade; Prognóstico.

\section{INTRODUÇÃO}

Cerca de $30 \%$ das mortes por trauma estão relacionadas à ação dos projéteis de arma de fogo (FPAF) ou instrumentos perfurocortantes (FIPC) ${ }^{1}$. No Brasil, no ano de 1996, houve 55.457 mortes por causas externas, entre elas 38.894 por agressões, 6.743 secundárias à lesões provocadas voluntariamente e 9.820 por eventos cuja intenção não foi determinada ${ }^{2}$.

A hemorragia interna grave é uma causa freqüente de morte neste grupo de doentes ${ }^{3}$. Os ferimentos penetrantes de tronco podem ser rapidamente letais, secundários à

1. Médico Assistente do Serviço de Emergência

2. Professor Assistente - Doutor.

3. Professor Titular. Diretor do Serviço de Emergência.

Recebido em 29/05/2001

Aceito para publicação em 18/06/2002

Trabalho realizado no Serviço de Emergência do Departamento de Cirurgia da Faculdade de Ciências Médicas da Santa Casa de São Paulo. 
hemorragia resultante de lesões cardíacas, hepáticas, esplênicas, renais e de grandes vasos. Nos traumatizados, a exsanguinação está envolvida em $44 \%$ das mortes pré-hospitalares e $55 \%$ das mortes após a admissão hospitalar ${ }^{3}$.

Houve mudanças consideráveis no tratamento operatório da exsanguinação. Durante muito tempo, o tratamento definitivo de todas as lesões encontradas na operação era a regra. Contudo, freqüentemente a agressão operatória ultrapassava as reservas fisiológicas, o choque hemorrágico alcançava sua fase irreversível e o doente falecia durante ou logo após a operação. Atualmente sabese que nesta situação a única maneira de mudar o prognóstico é interromper este ciclo. O termo damage control, ou "controle de danos", surgiu em 1993, proposto por Rotondo et al., definindo a conduta de interromper a laparotomia na presença de acidose, coagulopatia e hipotermia, através de um controle parcial da hemorragia e contaminação, com reoperação programada ${ }^{4}$. Portanto, o controle de danos é constituído de três fases: a laparotomia abreviada, a recuperação dos parâmetros fisiológicos na unidade de terapia intensiva e a reoperação programada. Atualmente sua aplicação ultrapassa os limites do trauma abdominal, sendo utilizado também para o tratamento de lesões torácicas, ortopédicas e mesmo no trauma vascular de extremidades ${ }^{5-7}$.

Entretanto, não é um procedimento isento de complicações. A persistência do sangramento e reoperação precoce para revisão da hemostasia podem ser necessários em até $15 \%$ dos $\operatorname{casos}^{8}$. A freqüência de peritonite e abscessos intra-abdominais oscila entre $12 \%$ e $67 \%$. A indicação do controle de danos deveria ser sustentada por critérios objetivos. Contudo, baseia-se principalmente em experiência pessoal, o que dificulta a identificação precoce dos candidatos. Também não é fácil definir-se o momento exato para a interrupção da operação. Acidose metabólica, hipotermia e coagulopatia são termos qualitativos, que abrangem uma ampla gama de valores, bem como inúmeras situações clínicas que podem ou não constituir casos ideais para operações abreviadas. É importante salientar que a indicação do damage control não deve ser resumida a apenas uma alternativa no final de uma laparotomia malsucedida ${ }^{10}$.

Considerando que os traumatizados com alta probabilidade de morrer devido à hemorragia são os que mais se beneficiariam desta tática, a identificação de variáveis relacionadas à hemorragia letal constitui uma maneira efetiva e precoce para o reconhecimento dos candidatos ao damage control. A presença destes indicadores ratificaria a interrupção da operação para a recuperação fisiológica.

Tendo em vista as vantagens da identificação precoce dos traumatizados com alta probabilidade de falecer por hemorragia, este estudo tem como objetivo determinar os indicadores de hemorragia letal em vítimas de trauma penetrante de tronco, admitidas com hipotensão arterial sistêmica.

\section{MÉTODO}

Foram analisados os dados de 74 vítimas de ferimentos penetrantes exclusivamente de tronco, com idade superior a 13 anos, atendidas no Serviço de Emergência da Santa Casa de São Paulo entre dezembro de 1996 e dezembro de 1998, admitidas com hipotensão arterial sistêmica secundária à hemorragia, que sobreviveram até o tratamento definitivo e nos quais não foi empregado o "controle de danos".

Foram considerados penetrantes os ferimentos que violaram a pleura parietal no tórax, peritônio parietal na parede abdominal anterolateral ou musculatura na região lombar. O "tronco" foi definido por limites anatômicos. $\mathrm{Na}$ região anterior, as clavículas constituíram seu limite superior e os ligamentos inguinais, o inferior. Posteriormente estendia-se desde o músculo trapézio superiormente até as pregas glúteas inferiormente. Hipotensão arterial sistêmica foi definida como pressão arterial sistólica menor ou igual a $90 \mathrm{mmHg}$.

No período em questão, foram coletados prospectivamente os dados de 2.330 traumatizados atendidos no Serviço de Emergência da Santa Casa de São Paulo. O trauma penetrante ocorreu em 537 e, destes, 116 foram admitidos com hipotensão arterial sistêmica. Cento e quatro apresentavam ferimentos penetrantes no tronco. Destes doentes, foram excluídos os que apresentaram lesões em extremidades ou região cefálica com Abbreviated Injury Scale (AIS) ${ }^{11}$ $>1$, os com causas não hemorrágicas para a hipotensão arterial sistêmica à admissão, os que não apresentavam sinais vitais à admissão ou que faleceram antes do tratamento (laparotomia ou toracotomia), os que foram submetidos à toracotomia para reanimação na sala de admissão e os submetidos a técnicas de controle de danos. Conforme estes critérios, foram excluídos 30 doentes e a amostra do estudo composta pelos 74 casos restantes.

A média etária variou entre 16 e 52 anos, com média de $29,5 \pm 8$ anos, sendo 62 doentes $(83 \%)$ do sexo masculino. Trinta e nove $(52 \%)$ foram vítimas de ferimentos por instrumentos perfurocortantes (FIPC) e 35 $(47 \%)$ sofreram traumatismo por ferimentos por projéteis de arma de fogo (FPAF).

Foram avaliadas informações a respeito do mecanismo do trauma, forma de transporte até o hospital, dados vitais, exame físico e tratamento à admissão. Calcularam-se os índices de trauma fisiológicos Revised Trauma Score (RTS) e a Escala de Coma de Glasgow (ECG) ${ }^{12,13}$.

Calculamos o Injury Severity Score (ISS), o Penetrating Abdominal Trauma Index (PATI), o Penetrating Thoracic Trauma Index (PTTI) e o Penetrating Trauma Index (PTI) para a estratificação da gravidade da amostra, além da probabilidade de sobrevivência pelo método TRISS ${ }^{14-17}$. Consideramos "mortes evitáveis" as que ocorreram em doentes com TRISS superior a $75 \%$.

Para a identificação dos indicadores de hemorragia letal, os doentes foram separados em dois grupos. O grupo $\mathrm{H}$ foi composto pelos que faleceram devido à hemorragia. $\mathrm{O}$ restante constituiu o grupo $\mathrm{O}$. A comparação das variáveis entre estes dois grupos estabeleceu quais as significativamente associadas à hemorragia letal. A análise estatística foi realizada através do software Statistical Package for Social Sciences (SPSS)®. Considerando-se valores de $\mathrm{p} \leq 0,05$ como significativos. 
As variáveis comparadas entre os grupos foram idade, mecanismo de trauma, localização do ferimento, tempo entre o trauma e admissão, dados fisiológicos à admissão, índices de trauma fisiológicos, reposição endovenosa de líquidos à admissão, recuperação da pressão arterial sistólica após a reposição volêmica, tempo operatório, pressão arterial sistólica no início e final da operação, $\mathrm{pH}$ e consumo de base no início e final da operação, reposição endovenosa de líquidos intra-operatória, órgãos lesados e índices anatômicos de trauma.

Para os dados numéricos foi aplicado inicialmente o teste $t$ de Student, controlado pelo teste de Levene. As variáveis com diferença significativa tiveram seus valores estratificados aleatoriamente em intervalos. Seguiu-se a identificação do primeiro intervalo que atingiu significado estatístico ( $\mathrm{p} \leq 0,05)$ através da correlação de Spearman, sendo este valor denominado "valor de corte". Portanto, a partir do "valor de corte", houve aumento significativo no número de mortes por hemorragia. $\mathrm{O}$ valor preditivo positivo e negativo para hemorragia letal foi calculado para cada valor de corte. Para análise das variáveis nominais foi aplicada a correlação de Spearman.

As variáveis aplicáveis em toda a amostra foram submetidas a análise multivariada através da correlação de Spearman. Os coeficientes de Pearson e Spearman foram empregados para a quantificação da correlação entre as variáveis. Construiu-se um modelo de regressão logística através do método "Foward", no qual a probabilidade de inclusão ou exclusão das variáveis para cada passo foi estipulada em $1 \%$.

\section{RESULTADOS}

O tempo entre o trauma e a admissão variou de 15 a 60 minutos, com média de $20 \pm 11$ minutos. Os dados vitais e índices de trauma à admissão estão expostos na Tabela 1. A infusão de volume à admissão variou entre
500 e $4.500 \mathrm{ml}$, com média de 2212,3 $\pm 660 \mathrm{ml}$. Dados em relação à estabilização hemodinâmica à reposição foram registrados em 65 casos. Quarenta e nove (75\%) apresentaram estabilização hemodinâmica após a reposição.

Houve 18 traumatizados com ferimentos em tórax, 25 com ferimentos na transição tóraco-abdominal, 19 com ferimentos em abdome e 12 com ferimentos múltiplos. Quarenta e nove (66\%) doentes foram submetidos à laparotomia com ou sem drenagem de tórax associada. Dezessete $(23 \%)$ foram submetidos à toracotomia, com ou sem drenagem de tórax contralateral. Três traumatizados (4\%) foram submetidos tanto à laparotomia quanto à toracotomia. Cinco doentes (6\%) não necessitaram laparotomia ou toracotomia, e dois foram submetidos à toracoscopia e os outros três somente à drenagem de tórax. O tempo operatório variou entre 30 e 370 minutos, com média de 176,2 \pm 89 minutos. $\mathrm{O}$ volume total de líquidos infundidos durante a operação variou de 1.500 a $31.700 \mathrm{ml}$, com média de $7.598,5 \pm 5.830 \mathrm{ml}$. O volume de concentrados de hemácias reposto esteve entre 0 a $5.400 \mathrm{ml}$, com média de $1.414,9 \pm 1.265 \mathrm{ml}$.

Cinqüenta e dois doentes $(70 \%)$ apresentavam lesões de vísceras torácicas, com AIS entre 2 e 5, com média de 3,6 $\pm 0,9$. Quarenta e nove traumatizados $(66,2 \%)$ tinham lesões em vísceras abdominais, com AIS entre 2 e 5 , e média de 3,65 $\pm 0,8$. Lesões tanto em vísceras torácicas como abdominais foram encontradas em 27 casos (36,5\%). A relação dos órgãos lesados está apresentada na Tabela 2.

O ISS foi calculado nos 74 doentes, variou entre $4 \mathrm{e}$ 54 , com média de 18,8 \pm 8 . O PATI, calculado nas 22 vítimas com lesões somente em vísceras abdominais, oscilou entre 4 e 54 , com média de 28,6 \pm 14 . O PTTI nas 25 vítimas com lesões somente em vísceras torácicas, alcançou limites de 4 e 25, com média de 13,1 \pm 6 . Nas 27 vítimas com trauma em vísceras torácicas e abdominais concomitantes, o PTI variou entre 12 e 51, com média de $29,3 \pm 12$. A média dos valores do TRISS foi $80,2 \pm 30 \%$, com limites de $2 \%$ e $100 \%$.

Tabela 1

Valor máximo, mínimo e média e desvio padrão dos dados fisiológicos à admissão

\begin{tabular}{|c|c|c|c|c|c|}
\hline Variável & Mínimo & Máximo & \multicolumn{3}{|c|}{ Média \pm Desvio Padrão } \\
\hline Pressão arterial sistólica & Inaudível & $90 \mathrm{mmHg}$ & 47,9 & \pm & $37 \mathrm{mmHg}$ \\
\hline Freqüência cardíaca & $60 \mathrm{bpm}$ & $160 \mathrm{bpm}$ & 115 & & $19 \mathrm{bpm}$ \\
\hline Freqüência respiratória & Zero & $32 \mathrm{ipm}$ & 24,8 & \pm & 9ipm \\
\hline Escala de Coma de Glasgow & 3 & 15 & 12 & \pm & 3 \\
\hline T-RTS & 1 & 12 & 8,6 & \pm & 3 \\
\hline RTS & 0,29 & 7,8408 & 5,403 & \pm & 2,138 \\
\hline
\end{tabular}

bpm : batimentos cardíacos por minuto.

Ipm : incursões respiratórias por minuto.

T-RTS: Revised Trauma Score (triagem)

RTS: Revised Trauma Score 
Tabela 2

Órgãos lesados

\begin{tabular}{|c|c|c|c|}
\hline & Órgão & & $\begin{array}{l}\text { mero de } \\
\text { ntes (\%) }\end{array}$ \\
\hline Tórax & Pulmão & & $(44,6 \%)$ \\
\hline & Coração & 13 & $(17,5 \%)$ \\
\hline & Grandes vasos ${ }^{1}$ & 4 & $(5,4 \%)$ \\
\hline & Vasos da parede torácica ${ }^{2}$ & 3 & $(4 \%)$ \\
\hline & Diafragma & 22 & $(29,7 \%)$ \\
\hline Abdome & Fígado & 29 & $(39,1 \%)$ \\
\hline & Cólon & 16 & $(21,6 \%)$ \\
\hline & $\begin{array}{l}\text { Intestino delgado } \\
\quad \text { (exceto duodeno) }\end{array}$ & 15 & $(20,2 \%)$ \\
\hline & Vasos maiores $^{3}$ & 11 & $(14,9 \%)$ \\
\hline & Estômago & 10 & $(13,5 \%)$ \\
\hline & Baço & 9 & $(12,1 \%)$ \\
\hline & Rim & 6 & $(8,1 \%)$ \\
\hline & Duodeno & 6 & $(8,1 \%)$ \\
\hline & Pâncreas & 5 & $(6,7 \%)$ \\
\hline & Bexiga & 1 & $(1,3 \%)$ \\
\hline & Ovário & 1 & $(1,3 \%)$ \\
\hline
\end{tabular}

1. Vasos do hilo pulmonar ou ${ }^{a}$ Subclávia.

2. Vasos Intercostais ou Artéria Mamária Interna.

3. Vasos Ilíacos Comuns, Vasos Ilíacos Internos, ou Vasos Ilíacos Externos, Veia Cava Inferior, Veia Porta, Artéria Hepática, Veia Renal ou Veia Mesentérica Superior.

Dos 61 doentes que sobreviveram à operação, 31 $(50,8 \%)$ apresentaram 67 complicações, sendo as mais frequientes o choque refratário $(13,1 \%)$, a hipotermia $(10,9 \%)$, e pneumonia $(10,9 \%)$. Houve 23 óbitos $(31 \%)$, 13 intra-operatórios e 10 após o término da operação (Tabela 3$)$. Dezenove $(82,6 \%)$ foram devido à hemorragia, englobando os 13 óbitos intra-operatórios e seis pós-operatórios, todos até 24 horas após a operação (Tabela 4). Houve 11 óbitos evitáveis, correspondendo a $47,8 \%$ das mortes. Dos 19 óbitos por hemorragia, oito (42\%) foram considerados evitáveis.

A comparação das variáveis entre os grupos $\mathrm{He} \mathrm{O}$ para a identificação dos indicadores de hemorragia letal está exposto nas Tabelas 5, 6, 7, 8, 9.

As variáveis tempo entre o trauma e admissão, pressão arterial sistólica à admissão, ECG, T-RTS e RTS à admissão foram significativamente diferentes entre os grupos, o que não ocorreu na avaliação do mecanismo de trauma e volume infundido à admissão (Tabelas 5 e 6). A letalidade por hemorragia esteve associada à persistência da hipotensão arterial sistêmica após a reposição de fluidos à admissão (Tabela 7).
Tabela 3

Causas dos 23 óbitos da amostra

\begin{tabular}{l|cc}
\hline Causa de óbito & Número $(\%)$ \\
\hline Hemorragia & $19 \quad(82,6 \%)$ \\
Síndrome da angústia respiratória do adulto & 3 & $(13 \%)$ \\
Falência de múltiplos órgãos e sistemas & 1 & $(4,3 \%)$ \\
\hline Total & $23(100 \%)$ \\
\hline
\end{tabular}

Tabela 4

Principal foco de sangramento responsável pelo óbito de 19 traumatizados

\begin{tabular}{l|cc}
\hline Foco de sangramento & \multicolumn{2}{c}{ Número (\%) } \\
\hline Vasos abdominais $^{\mathrm{a}}$ & 6 & $(31,5 \%)$ \\
Coração & 4 & $(21 \%)$ \\
Vasos do hilo pulmonar & 3 & $(15,8 \%)$ \\
Fígado & 2 & $(10,5 \%)$ \\
Mais de um foco de sangramento & 4 & $(21 \%)$ \\
\hline Total & 19 & $(100 \%)$ \\
\hline
\end{tabular}

${ }^{a}$ Veia Ilíaca Comum, Artéria Ilíaca Externa, Veia Ilíaca Externa, Veia Porta e Veia Cava Inferior.

Ao início da operação, tanto a pressão arterial sistólica como o $\mathrm{pH}$ e excesso de base (EB) foram significativamente diferentes entre os grupos (Tabela 5). Isto se repetiu ao término da operação para a pressão arterial sistólica e $\mathrm{pH}$.

As vítimas de lesões somente em vísceras abdominais apresentaram letalidade por hemorragia maior (41\%), seguidas das vítimas de lesões somente em vísceras torácicas $(24 \%)$ e dos traumatizados com lesões tanto em vísceras torácicas como abdominais (14\%), embora a diferença não tenha sido significativa $(\mathrm{p}=0,4)$ (Tabela 8$)$.

A letalidade por hemorragia dos doentes com lesões vasculares com AIS > $2(60 \%)$ foi maior que a observada para doentes com lesões cardíacas com AIS $>2(33 \%)$ e portadores de lesões em fígado, baço ou rim com AIS > 2 (14\%) $(\mathrm{p}=0,007)$ (Tabela 9). As médias dos ISS, PATI, PTTI dos traumatizados que faleceram por hemorragia foram significativamente maiores (Tabela 5).

Os valores preditivos positivos e negativos para cada variável e valor de corte estão expostos na Tabela 10.

A Tabela 11 expõe a relação das variáveis com a hemorragia letal, quando estudadas pelo método da análise multivariada através do cálculo da correlação de Spearman. Foram incluídas neste estudo as que demons- 
Tabela 5

Comparação das variáveis numéricas entre os grupos $\mathrm{H}$ e $\mathrm{O}$

\begin{tabular}{|c|c|c|c|c|c|}
\hline Variável & & $H$ & & $O$ & $p$ \\
\hline Tempo trauma-admissão & 25,3 & $12 \mathrm{~min}$. & 18,2 & $\pm 10 \mathrm{~min}$ & 0,033 \\
\hline PAS à admissão & 25,3 & $35 \mathrm{mmHg}$ & 55,8 & $\pm 34 \mathrm{mmHg}$ & 0,002 \\
\hline Escala de Coma de Glasgow & 10,6 & \pm & 13,5 & \pm & $<0,001$ \\
\hline T-RTS & 6,9 & \pm & 9,3 & \pm & $<0,001$ \\
\hline RTS & 3,8907 & 2,542 & 5,9254 & 1,716 & $<0,001$ \\
\hline Volume à admissão & 2123,5 & $\pm 695 \mathrm{ml}$ & 2243,8 & $\pm 651 \mathrm{ml}$ & 0,52 \\
\hline PAS início da operação & 48,8 & $40 \mathrm{mmHg}$ & 106,8 & $\pm 22 \mathrm{mmHg}$ & $<0,001$ \\
\hline pH início da operação & 6,99 & 0,17 & 7,24 & 0,9 & $<0,001$ \\
\hline EB início operação & $-16,1$ & \pm & $-8,5$ & \pm & $<0,001$ \\
\hline PAS final da operação * & 50 & $16 \mathrm{mmHg}$ & 102 & $\pm 16 \mathrm{mmHg}$ & $<0,001$ \\
\hline pH final da operação * & 7,14 & 0,4 & 7,31 & 0,8 & $<0,001$ \\
\hline Volume intra-operatório & 10950 & $\pm 7008 \mathrm{ml}$ & 6547 & $\pm 5041 \mathrm{ml}$ & 0,007 \\
\hline Conc. hemácias intra-operatório & 2418 & \pm 1615 & 1100 & \pm 951 & $<0,001$ \\
\hline Tempo operatório * & 312 & $\pm 92 \mathrm{~min}$. & 186 & $\pm 70 \mathrm{~min}$ & $<0,001$ \\
\hline ISS & 23,4 & \pm & 17,3 & \pm & 0,01 \\
\hline PATI & 36,7 & 12 & 22,3 & \pm 11 & 0,015 \\
\hline PTTI & 20,5 & \pm & 12,6 & \pm & 0,001 \\
\hline
\end{tabular}

* Excluíndo-se os que faleceram no intra-operatório, PAS: Pressão arterial sistólica, RTS: Revised Trauma Score, EB: Excesso de base, ISS: Injury Severity Score, PATI: Penetrating Abdominal Trauma Index, PTTI: Penetrating Thoracic Trauma Index.

Tabela 6

Comparação do mecanismo de trauma entre os grupos $(\mathrm{p}=0,54)$

\begin{tabular}{l|c|c|c}
\hline Grupo & Número total & $F P A F^{a}$ & FIPC $^{b}$ \\
\hline $\mathrm{H}$ & 19 & 10 & 9 \\
$\mathrm{O}$ & 55 & 25 & 30 \\
\hline Total & 74 & 35 & 39 \\
\hline
\end{tabular}

${ }^{a}$ Número de vítimas com ferimentos por projéteis de arma de fogo ${ }^{b}$ Número de vítimas com ferimentos por instrumentos perfurocortantes.

traram melhor relação com hemorragia letal na análise univariada, com exceção do PATI, do PTTI, da pressão arterial sistólica ao final da operação por serem aplicáveis somente em grupos específicos de doentes. Os maiores valores absolutos dos coeficientes correlação, que quantificam a relação entre as variáveis, foram atribuídos a pressão arterial sistólica no início da operação, $\mathrm{pH}$ arterial no início da operação e estabilização da pressão arterial sistólica após a reposição volêmica à admissão.

Incluindo-se todas as variáveis acima, o modelo de regressão logística foi capaz de encontrar uma função para cálculo da probabilidade de hemorragia letal, baseada nas variáveis pressão arterial sistólica no início da operação e
Tabela 7

Comparação do número de doentes que alcançaram estabilidade hemodinâmica após reposição hídrica entre os grupos $(\mathrm{p}<0,001)$

\begin{tabular}{lcccc}
\hline Grupos & Número total & Estáveis $^{a}$ & Instáveis $^{b}$ \\
\hline $\mathrm{H}$ & 16 & 6 & 10 \\
$\mathrm{O}$ & 49 & 43 & 6 \\
\hline Total & 65 & 49 & 16 \\
\hline
\end{tabular}

${ }^{a}$ Doentes com pressão arterial sistólica maior que $90 \mathrm{mmHg}$ após reposição.

${ }^{b}$ Doentes com pressão arterial sistólica igual ou menor que $90 \mathrm{mmHg}$ após a reposição.

volume de concentrados de hemácias transfundidos durante a operação, descrita abaixo.

Probabilidade de hemorragia letal $=1 / 1+\mathrm{e}^{-\mathrm{b}}$

Onde:

$\mathrm{e}=2,718282$

$\mathrm{b}=0,0399$ PASIO $+0,0008 \mathrm{CH}$

PASIO: Pressão arterial sistólica no início da operação, em $\mathrm{mmHg}$.

$\mathrm{CH}$ : Volume de concentrado de hemácias, em mililitros, transfundido durante a operação. 
Tabela 8

Comparação da frequiência de lesões em vísceras abdominais, torácicas e em ambos segmentos corporais entre os grupos $(\mathrm{p}=0,4)$

\begin{tabular}{l|c|c|c|c}
\hline Grupo & Número total & $A^{a}$ & $T^{b}$ & $T+A^{c}$ \\
\hline H & 19 & 9 & 6 & 4 \\
O & 55 & 13 & 19 & 23 \\
\hline Total & 74 & 22 & 25 & 27 \\
\hline
\end{tabular}

${ }^{a}$ Número de doentes com lesões somente em vísceras abdominais (A).

${ }^{b}$ Número de doentes com lesões somente em vísceras torácicas $(T)$. ${ }^{c}$ Número de doentes com lesões em ambos segmentos corporais $(T+A)$.
Tabela 9

Comparação da freqüência de lesões com AIS > 2 em vasos, coração e outros órgãos entre os grupos $(\mathrm{P}=0,007)$

\begin{tabular}{l|c|c|c|c}
\hline Grupo & Número total & Vasos $^{a}$ & Coração $^{b}$ & Outros $^{c}$ \\
\hline $\mathrm{H}$ & 15 & 9 & 4 & 2 \\
$\mathrm{O}$ & 32 & 6 & 8 & 18 \\
\hline Total & 47 & 15 & 12 & 20 \\
\hline
\end{tabular}

${ }^{a}$ Número de doentes com lesões com AIS>2 em vasos somente.

${ }^{b}$ Número de doentes com lesões com AIS $>2$ em coração somente.

${ }^{c}$ Número de doentes com lesões com AIS $>2$ em outros órgãos, excluindo-se coração e vasos.

Tabela 10

Valores preditivos positivos e negativos para os valores de corte associados a cada variável

\begin{tabular}{|c|c|c|c|}
\hline Variável & $V P P$ & $V P N$ & $O . R$. \\
\hline Instabilidade hemodinâmica após reposição volêmica & $62 \%$ & $87 \%$ & 11,9 \\
\hline Tempo entre o trauma e admissão $\geq 30$ minutos & $46 \%$ & $78 \%$ & 3,0 \\
\hline Pressão arterial sistólica à admissão $\leq 70 \mathrm{mmHg}$ & $34 \%$ & $88 \%$ & 4,1 \\
\hline Escala de Coma de Glasgow $\leq 10$ & $54 \%$ & $80 \%$ & 4,7 \\
\hline $\mathrm{T}-\mathrm{RTS} \leq 7$ & $33 \%$ & $88 \%$ & 3,8 \\
\hline $\mathrm{RTS} \leq 6,5$ & $33 \%$ & $88 \%$ & 3,8 \\
\hline PAS no início da operação $\leq 110 \mathrm{~mm} \mathrm{Hg}$ & $37 \%$ & $100 \%$ & 12,6 \\
\hline $\mathrm{pH} \leq 7,25$ no início da operação & $19 \%$ & $100 \%$ & 4,3 \\
\hline $\mathrm{EB} \leq-10 \mathrm{mEq} / \mathrm{L}$ no Início da operação & $23 \%$ & $97 \%$ & 8,2 \\
\hline PAS no final da operação $\leq 100 \mathrm{mmHg}$ & $17 \%$ & $100 \%$ & 4,3 \\
\hline $\mathrm{pH} \leq 7,25$ no final da operação & $33 \%$ & $37 \%$ & 17,5 \\
\hline Infusão líquidos intra-operatória $\geq 8.000 \mathrm{ml}$ & $42 \%$ & $85 \%$ & 4,2 \\
\hline Infusão c. hemácias intra-operatória $\geq 1.200 \mathrm{ml}$ & $37 \%$ & $90 \%$ & 5,5 \\
\hline Lesão vascular AIS > 2 & $50 \%$ & $80 \%$ & 4,3 \\
\hline PATI $\geq 22$ & $57 \%$ & $87 \%$ & 9,3 \\
\hline PTTI $\geq 15$ & $40 \%$ & $100 \%$ & 6,6 \\
\hline Tempo operatório $\geq 250$ minutos & $33 \%$ & $97 \%$ & 21,0 \\
\hline
\end{tabular}

O.R.: Odds Ratio, VPP: valor preditivo positivo, VPN: valor preditivo negativo, PAS: pressão arterial sistólica, EB: excesso de base, PATI: Penetrating Abdominal Trauma Index, PTTI: Penetrating Thoracic Trauma Index, AIS: Abbreviated Injury Scale, RTS: Revised Trauma Score.

Construiu-se uma matriz com valores aleatórios destas variáveis para facilitar o emprego desta função (Tabela 12).

\section{DISCUSSÃO}

Provavelmente a melhora do atendimento pré-hospitalar, o aumento da violência urbana e dos acidentes automobilísticos são responsáveis pela complexidade crescente dos traumatizados que alcançam serviço hospitalar. Aqueles que antes faleciam no local do trauma passaram a ser admitidos em centros especializados e as situações extremas tornaram-se mais frequientes para o cirurgião.

A exsanguinação tem um papel muito importante neste contexto. É considerada a segunda causa de mortes 
Tabela 11

Relação das variáveis estudadas com a hemorragia letal, segundo a análise multivariada pelo cálculo da correlação de Spearman e coeficiente de correlação

\begin{tabular}{|c|c|c|}
\hline Variável & $p$ & $\begin{array}{c}\text { Coefic. de } \\
\text { correlação* }\end{array}$ \\
\hline PAS no início da operação & $<0,001$ & $-0,6776$ \\
\hline pH arterial no início da operação & $<0,001$ & $-0,6163$ \\
\hline Instabilidade hemodinâmica após reposição volêmica & $<0,001$ & $-0,5751$ \\
\hline $\mathrm{pH}$ arterial no final da operação & 0,005 & $-0,5105$ \\
\hline Volume de conc. de hemácias no intra-operatório & $<0,001$ & $+0,4478$ \\
\hline Excesso de base no início da operação & 0,004 & $-0,4352$ \\
\hline Revised Trauma Score & 0,001 & $-0,4185$ \\
\hline Escala de Coma de Glasgow & $<0,001$ & $-0,4074$ \\
\hline Lesão vascular com AIS > 2 & $<0,001$ & $+0,3962$ \\
\hline PAS à admissão & 0,001 & $-0,3602$ \\
\hline Volume total infundido durante a operação & 0,004 & $+0,3244$ \\
\hline Tempo Trauma - admissão & 0,033 & $+0,2906$ \\
\hline
\end{tabular}

* Para as variáveis numéricas utilizou-se o coeficiente de Pearson e para as nominais o de Spearman.

PAS: Pressão arterial sistólica, AIS: Abbreviated Injury Scale.

Tabela 12

Probabilidade de hemorragia letal, em \%, calculada com base na pressão arterial sistólica aferida no início da operação e o volume de concentrado de hemácias infundido durante a operação

\begin{tabular}{|c|c|c|c|c|c|c|c|c|c|c|c|c|}
\hline$P A C H$ & 900 & 1.200 & 1.500 & 1.800 & 2.100 & 2.400 & 2.700 & 3.000 & 3.300 & 3.600 & 3.900 & 4.200 \\
\hline 0 & 67 & 72 & 77 & 81 & 84 & 87 & 90 & 92 & 93 & 95 & 96 & 97 \\
\hline 10 & 58 & 64 & 69 & 74 & 78 & 82 & 85 & 88 & 90 & 92 & 94 & 95 \\
\hline 20 & 48 & 54 & 60 & 66 & 71 & 75 & 80 & 83 & 86 & 89 & 91 & 93 \\
\hline 30 & 38 & 44 & 50 & 56 & 62 & 67 & 72 & 77 & 81 & 84 & 87 & 90 \\
\hline 40 & 29 & 35 & 40 & 46 & 52 & 58 & 64 & 69 & 74 & 78 & 82 & 85 \\
\hline 50 & 22 & 26 & 31 & 36 & 42 & 48 & 54 & 60 & 66 & 71 & 75 & 80 \\
\hline 60 & 16 & 19 & 23 & 28 & 33 & 38 & 44 & 50 & 56 & 62 & 67 & 72 \\
\hline 70 & 11 & 14 & 17 & 21 & 25 & 29 & 35 & 40 & 46 & 52 & 58 & 64 \\
\hline 80 & 8 & 10 & 12 & 15 & 18 & 22 & 26 & 31 & 37 & 42 & 48 & 54 \\
\hline 90 & 5 & 7 & 8 & 10 & 13 & 16 & 19 & 23 & 28 & 33 & 38 & 44 \\
\hline 100 & 4 & 5 & 6 & 7 & 9 & 11 & 14 & 17 & 21 & 25 & 30 & 35 \\
\hline 110 & 2 & 3 & 4 & 5 & 6 & 8 & 10 & 12 & 15 & 18 & 22 & 26 \\
\hline
\end{tabular}

CH : Volume de concentrado de hemácias transfundido, em mililitros.

PA: Pressão arterial sistólica aferida no início da operação, em mmHg.

em traumatizados, superada apenas pelo trauma craniencefálico, e é o principal fator envolvido nas mortes até 48 horas do traumatismo ${ }^{3,18}$. Há uma estreita relação entre exsanguinação e trauma penetrante, tanto pela freqüência com que estão associados quanto pelo prognóstico que encerram. Cerca de $95 \%$ das mortes hospitalares após trauma penetrante ocorrem nas primeiras 48 horas da admissão, freqüentemente devido à hemorragia ${ }^{3}$. Quanto mais 
rapidamente a hemorragia for controlada, melhor é o prognóstico. O objetivo do nosso estudo visa justamente a identificação precoce destes traumatizados altamente suscetíveis à hemorragia potencialmente letal.

Na nossa análise, o mecanismo de trauma não apresentou relação com a letalidade por hemorragia. Razuk, em 1999, não encontrou relação desta variável com a letalidade nas vítimas de ferimentos cardíacos penetrantes ${ }^{19}$. Todavia, Coimbra et al., em 1995, e Asensio et al., em 1998, relataram que o trauma causado por projéteis de armas de fogo foi um fator prognóstico independente nas vítimas de ferimentos cardíacos 20,21 .

Notamos que um intervalo de tempo entre o trauma e a admissão acima de 30 minutos foi um fator significativo para a ocorrência de hemorragia letal. Quando analisamos o volume de cristalóides ou concentrados de hemácias infundidos no período pré-operatório, não notamos influência na frequiência de hemorragia letal. Estas evidências sugerem que o tempo para o controle do foco de sangramento, mais que a própria reposição hídrica préoperatória, deve ser valorizado nas vítimas de trauma penetrante em choque.

Há vários relatos de que o "choque "ou "hipotensão arterial" é um marcador de mau prognóstico em trauma ${ }^{22,23}$. Nossos dados sugerem que há uma estratificação da gravidade. Nas vítimas de trauma penetrante, não se deve interpretar os "hipotensos" como um grupo homogêneo. Indicadores de hemorragia letal foram reconhecidos, o que nos permite a identificação precoce dos mais propensos a esta evolução.

O Revised Trauma Score teve alta correlação com a letalidade por hemorragia. Certamente, o T-RTS é o índice mais prático para o cálculo. Com três variáveis de fácil memorização, representa um bom indicador de hemorragia letal.

Embora a reposição endovenosa de líquidos à admissão não tenha tido relação com a letalidade por hemorragia, a resposta hemodinâmica à mesma constituiu um fator importante na evolução. A incapacidade de atingir a estabilidade hemodinâmica após a reposição inicial caracteriza os doentes com alta probabilidade de morrer por hemorragia, mesmo que haja uma resposta parcial à infusão inicial. É o alerta mais importante do sangramento ativo, e esforços devem ser direcionados para diminuição do tempo até a hemostasia definitiva.

Observamos que os traumatizados com lesões somente em órgãos abdominais apresentaram letalidade por hemorragia maior, quando comparados aos que sustentavam lesões somente em vísceras torácicas ou em ambos segmentos corporais, porém sem significado estatístico. Provavelmente isto é um reflexo da freqüência de lesões vasculares abdominais, que apresentaram alta tendência à hemorragia letal. O envolvimento em mais de um segmento corporal não foi acompanhado de maior letalidade. Talvez isto ocorra pela lesão de várias vísceras que resultem em hemorragia de baixo fluxo, levando à hipotensão arterial sistêmica pelo volume total de sangue perdido, e que representam menor ameaça à vida pois o controle operatório é mais fácil.
A exsanguinação está associada principalmente aos ferimentos cardíacos, em grandes vasos abdominais ou torácicos e em vísceras maciças ${ }^{24}$. Em nosso estudo, cerca de 25\% (13/52) dos traumatizados com lesões em órgãos torácicos apresentavam ferimento cardíaco. Avaliando doentes com lesões em vísceras abdominais, 28,5\% (14/49) sustentavam ferimento vascular maior e 59,1\% (29/49), traumatismo hepático.

Cerca de $80 \%$ dos traumatizados com ferimentos cardíacos morrem no local do trauma ou durante o transporte $^{25}$. Nos que alcançam o hospital, a letalidade oscila entre $25 \%$ e $70 \%$ e depende muito da amostra analisa$\mathrm{da}^{19-21}$. Em nosso estudo excluímos causas não hemorrágicas para hipotensão arterial sistêmica à admissão, inclusive o tamponamento cardíaco, e a letalidade por hemorragia associada aos ferimentos cardíacos foi $30 \%$.

Cerca de $35 \%$ a $80 \%$ dos traumatizados com lesões vasculares abdominais morrem ${ }^{26,27}$. Na nossa amostra, aproximadamente $54 \%$ dos traumatizados com ferimentos em grandes vasos abdominais faleceram por hemorragia.

A frequiência de hemorragia letal foi significativamente maior nos traumatizados com lesões de grandes vasos abdominais ou torácicos com AIS > 2, quando comparada à que apresentaram os doentes com lesões cardíacas com AIS > 2 e órgãos maciços com AIS > 2. De maneira ideal, esta avaliação deve ser feita entre doentes sustentando lesões com mesmo escore de gravidade, mas devido ao número reduzido de casos, analisamos como um todo o grupo com lesões graves (AIS > 2). Contudo é importante ressaltar que este é um fato diretamente relacionado à amostra analisada, e que está em função da presença ou não dos fatores prognósticos para cada órgão lesado.

A letalidade por hemorragia foi associada a $\mathrm{pH} \leq$ 7,25 e EB $\leq-10 \mathrm{mEq} / \mathrm{L}$ ao início da operação, o que também foi observado nos que persistiram com $\mathrm{pH}$ abaixo de 7,25 ao término da mesma. Uma das dificuldades na avaliação desta variável é o momento da coleta, especialmente após alguma reposição volêmica ou de bicarbonato. No estudo de Davis et al., em 1998, enquanto o excesso de base manteve relação com a letalidade até 48 horas após o trauma, o pH apresentou a mesma correlação somente até duas horas, o que foi explicado pela ação de outros sistemas tampão corrigindo o $\mathrm{pH}$, mesmo na presença de metabolismo anaeróbico ${ }^{28}$.

Em média, 7,6 litros de líquidos e 1,4 litro de concentrado de hemácias foram infundidos durante a operação. Contudo, a coagulopatia ocorre em $40 \%$ e a letalidade alcança $40 \%$ dos doentes submetidos a transfusões de mais de 10 unidades de concentrados de hemácias nas primeiras 24 horas $^{29}$.

Nossa análise demonstrou que a hemorragia letal foi associada à infusão intra-operatória de líquidos maior que 8 litros ou à transfusão de mais de $1.200 \mathrm{ml}$ de concentrados de hemácias. Não há dúvidas que quanto maior necessidade de infusão de líquidos intra-operatória pior é o prognóstico ${ }^{20,21}$. A depleção dos fatores coagulação, bem como o desenvolvimento de falência de múltiplos órgãos e sistemas, ocorre principalmente após a infusão de seis 
unidades de concentrados de hemácias ${ }^{30}$. O PATI e o PTTI foram desenvolvidos especificamente para o trauma penetrante, e foram considerados bons indicadores de hemorragia letal. Moore et al., em 1981, quando descreveram o PATI, relataram que valores acima de 25 associavam-se a um aumento na freqüência de complicações em mais de três vezes ${ }^{15}$. Na nossa análise, PATI $\geq 22$ ou PTTI $\geq 15$ se relacionaram com maior chance de hemorragia letal. O PTI foi empregado apenas nos 27 doentes que apresentavam lesões em mais de um segmento corporal, e, destes, quatro faleceram por hemorragia (14\%).

A letalidade da amostra por nós estudada alcançou $31 \%$, o que deve reforçar a preocupação com estes doentes. A maioria dos óbitos foi devida à hemorragia $(82 \%)$, ocorrendo até 24 horas após o trauma, como nos estudos de Sauaia et al., em 1995, e Gofrit et al., em $1997^{3,31}$. O foco da hemorragia letal foi atribuído a lesões cardíacas ou em grandes vasos em 68\%. Treze dos 19 óbitos por hemorragia ocorreram antes do término da operação, o que ressalta a importância do atendimento inicial e da hemostasia precoce. Todos os óbitos em vítimas de ferimentos cardíacos ocorreram durante a operação. Dos seis óbitos por hemorragia devido a ferimentos vasculares abdominais, quatro foram intra-operatórios. Alguns destes doentes apresentavam lesões graves e de difícil controle, como é o caso das vítimas de ferimentos cardíacos e dos doentes com lesões vasculares. Contudo, em certos casos pode ter havido tratamento inadequado das lesões e aumento desnecessário do tempo operatório, o que contribuiria para a persistência do sangramento e evolução com choque, hipotermia, coagulopatia e óbito.

Mesmo entre os 11 traumatizados que faleceram por hemorragia e possuíam TRISS inferior a 75\%, houve cinco casos em que a operação durou mais de duas horas, e, destes, três faleceram durante a operação.

Ainda analisando os casos de hemorragia letal em nossa amostra, notamos que técnicas para abreviar a operação permitindo a recuperação fisiológica na unidade de terapia intensiva (damage control) poderiam ter sido empregadas em pelo menos 10 doentes (52\%). O tempo operatório esteve acima de duas horas em oito destes casos.

Não é fácil decidir por técnicas para abreviar a operação. Freqüentes são os casos em que após exauridas as reservas fisiológicas do doente e frente à hipotermia coagulopatia e choque persistente, tenta-se o tamponamento da cavidade peritoneal com compressas como a última alternativa possível. Nesta situação os resultados são precários, pois a fase irreversível do choque hemorrágico já está instalada, e o óbito é apenas uma questão de tempo. Não há dúvida que a opção pelo emprego do damage control deve ser precoce, contudo devem existir limites para que esta técnica não seja utilizada indevidamente, o que resultaria em complicações.

Infelizmente, não há normas precisas disponíveis que auxiliem na decisão de interromper a operação. Vários autores tentaram definir as indicações para o damage control, mas ainda há pouca objetividade.

Carrillo et al., em 1993, baseavam sua decisão em critérios fisiológicos e na perda inicial de sangue ${ }^{32}$. Os doentes que apresentavam $\mathrm{pH}<7,25$, temperatura corporal central menor que $34^{\circ} \mathrm{C}$ ou tivessem uma perda inicial estimada maior que quatro litros de sangue deveriam ter sua laparotomia abreviada ${ }^{32}$. Feliciano et al., em 1996, consideraram que a indicação precisa para o damage control seria a acidemia persistente $(\mathrm{pH}<7,2)$ mesmo com o controle da hemorragia e reposição hídrica e eletrolítica adequadas ${ }^{33}$. Garrison et al., em 1996, avaliaram os traumatizados submetidos a laparotomias abreviadas e encontraram associação da letalidade com ISS > 35, tempo total de hipotensão maior que 70 minutos, tempo de protrombina maior que 19 segundos, tempo parcial de tromboplastina ativada maior de 60 segundos, número total acima de 15 unidades de concentrados de hemácias transfundido e $\mathrm{pH}<7,2^{34}$. Outros autores associaram os parâmetros acima para a definição da indicação $0^{35}$.

Hirshberg \& Walden, em 1997, ressaltaram que a presença das alterações fisiológicas ocorre com uma situação limítrofe já instalada, e que talvez esperar por estes sinais representaria perder o momento ideal para a indicação do damage control $^{36}$. Estes autores propõem que esta decisão seja baseada preferencialmente na avaliação da magnitude das lesões e no mecanismo de trauma.

A decisão por esta tática vai além dos detalhes técnicos. Depende da disponibilidade de vagas na Unidade de Terapia Intensiva e da equipe de cirurgiões responsável pelo acompanhamento minucioso do doente, preparada para intervir quando necessário.

Os indicadores de hemorragia letal relatados em nosso estudo podem ser considerados durante a decisão pelo damage control. A Tabela 10 demonstra os encontrados e seus valores preditivos em relação à hemorragia letal. O maior valor preditivo positivo foi $62 \%$, associado à ausência da estabilização hemodinâmica após a reposição volêmica à admissão, o que esteve presente em $24 \%$ da amostra. Portanto, se utilizássemos este parâmetro apenas, técnicas para abreviar a operação seriam utilizadas em 24\% dos nossos doentes. Mesmo assim, 13\% dos 76\% restantes faleceriam por hemorragia, uma vez que o valor preditivo negativo foi $87 \%$.

Krishna et al., em 1998, avaliaram os indicadores de letalidade nos traumatizados vítimas de exsanguinação submetidos a operações convencionais por trauma, quando operações abreviadas não foram realizadas ${ }^{37}$. Um estudo semelhante ao nosso, mas envolvendo doentes com mecanismo de trauma distintos. Através de regressão logística, propuseram um modelo que foi capaz de prever o prognóstico com até $92 \%$ de sensibilidade. As variáveis mais importantes foram a hipotermia (temperatura corporal central menor que $33^{\circ} \mathrm{C}$ ) e acidose metabólica grave $(\mathrm{EB}<-12 \mathrm{mEq} / \mathrm{L})$.

Em nosso estudo, todas as variáveis que foram submetidas à análise multivariada apresentaram relação independente com hemorragia letal. As que apresentaram maior correlação com mortes por hemorragia foram a pressão arterial sistólica e pH arterial no início da operação além do volume de concentrado de hemácias transfundi- 
do durante a operação. Portanto, já no início da operação é possível ter uma idéia razoável da evolução para hemorragia letal, através da avaliação da magnitude da lesão orgânica, pressão arterial sistólica e gasometria arterial.

A matriz com valores aleatórios calculada com base na equação de probabilidade de morte por hemorragia é de aplicação prática. Para determinado valor de pressão arterial sistólica aferida no início da operação, calcula-se o risco de hemorragia letal a cada unidade de concentrados de hemácias infundido. Desta forma, o cirurgião pode avaliar os riscos e benefícios, e decidir o momento ideal para interromper a operação.

Contudo, não é possível estabelecer regras fixas para o emprego do damage control. Trata-se do atendimento de traumatizados graves, quando a melhor opção muitas vezes não é equivalente à ideal, mas à que pode ser realizada. Nestes momentos, a experiência do cirurgião, sua capacidade de adaptação a situações adversas e sua criatividade para improvisar soluções são os fatores determinantes do sucesso.

\begin{abstract}
Objective: To identify predictors of death due to hemorrhage in patients sustaining penetrating trauma to the torso admitted in shock. Method: Data was prospectively collected from Dec 1996 until Dec 1998. Patients presenting penetrating wounds from clavicles to inguinal ligaments, admitted with a systolic blood pressure $(S B P)<90 m m H g$ were included. Those who died due to massive hemorrhage compounded group $H$ and the others, group $O$. Variables were compared between the groups. Statistical treatment involved a multivariate analysis with Spearman dispersion, considering $p<0.05$ as significant, and a logistic regression. Seventy-four patients met the inclusion criteria. There were 62 males (83\%), and the mean age was $29+8$ years. The mean RTS, ISS and TRISS were respectively $5.4+2.1,18+8$ and $80+30$. Nineteen died due to massive hemorrhage. Results: The main predictors of lethal hemorrhage were: persistent shock despite volume replacement, systolic bood pressure in the beginning of the operation $<110 \mathrm{mmHg}$, arterial $\mathrm{pH}<7.25$ in the beginning of operation, necessity of more than 1200ml of packed red blood cells during the operation and presence of vascular injury with AIS > 2. Conclusion: The risk of death due to hemorrhage can be assessed objectively. Based in this information, the selection of the patients for damage control becomes more objective and reliable.
\end{abstract}

Key Words: Penetrating trauma, Shock, Hemorrhagic shock, Mortality, Prognosis.

\title{
REFERÊNCIAS
}

1. Fingerhut LA. Epidemiology: Urban Mortality. In: Ivatury R. The textbook of penetrating trauma. Rose Tree Corporate Center, Media, EUA, Williams \& Wilkins, 1996, p.17-30.

2. Brasil. Ministério da Saúde. Datasus - Óbitos por ocorrência por causa - CID - BR, Capítulo XX, Causas externas de complicações e mortalidade, período de 1996. Disponível from internet <http:// www.datasus.gov.br/cg1/tabcg1.exe?sim/dybr.det.> [23 de março de 1999].

3. Sauaia A, Moore F, Moore E. Epidemiology of trauma deaths: a reassessment. J Trauma, 1995, 38: 185-92.

4. Rotondo M, Schwab WW, Mcgonival M et al. "Damage Control": An approach for improved survival in exsanguinating penetrating abdominal injury. J Trauma, 1993, 35: 375-82.

5. Henry SM, Tornetta P, Scalea T. Damage Control for devastating pelvic and extremity injuries. Surg Clin North Am, 1997, 77: 879-96.

6. Porter JM, Ivatury R, Nassoura ZE. Extending the horizons of "Damage Control" in unstable trauma patients beyond the abdomen and gastrointestinal tract. J Trauma, 1997, 42: 559-61.

7. Wall MJ, Soltero E. Damage control for thoracic injuries. Surg Clin North Am, 1997, 77: 863-78.
8. Morris JA, Eddy VA, Rutherford EJ. The trauma celiotomy: The evolving concepts of damage control. Curr Probl Surg, 1996, 33: 609-708.

9. Martin RR, Byrne M. Postoperative care and complications of damage control surgery. Surg Clin North Am, 1997, 77: 929-42.

10. Mattox KL. Introduction, background, and future projections of damage control surgery. Surg Clin North Am, 1997, 77: 753-9.

11. Association For The Advancement Of Automotive Medicine - The abbreviated injury scale - 1990 revision. Des Plaines, IL, 1990. 75p.

12. Champion HR, Sacco WJ, Copes WS et al. A revision of the trauma score. J Trauma, 1989, 29: 623-9.

13. Teasdale G, Jennet B. Assessment of coma and impaired consciuosness: a pratical scale. Lancet, 1974, 2: 81-4.

14. Baker S, O'Neill B, Haddon W et al. The injury severity score: a method for describing patients with multiple injury and evaluating emergency care. J Trauma, 1974, 14: 187-96.

15. Moore EE, Dunn E, Moore J et al. Penetrating abdominal trauma index. J Trauma, 1981, 21: 439-44.

16. Ivatury R, Nallathambi M, Sthal WM et al. Penetrating cardiac trauma: quantifying the severity of anatomic and physiologic injury. Ann Surg, 1987, 205: 61-6. 
17. Boyd CR, Tolson MA, Copes W. Evaluating trauma care: the TRISS method. J Trauma, 1987, 27: 370-8.

18. Shackford S, Mackersie RC, Holbrook TL et al. The epidemiology of traumatic death. Arch Surg, 1993, 128: 571-575.

19. Razuk Filho A. Ferimentos cardíacos penetrantes: Análise dos fatores relacionados à mortalidade. São Paulo, 1999. (Tese de Mestrado - Faculdade de Ciências Médicas da Santa Casa de São Paulo).

20. Coimbra R, Pinto MC, Razuk A et al. Penetrating cardiac wounds: predictive value of trauma indices and necessity of terminology standardization. Am Surg, 1995, 61: 448-52.

21. Asensio J, Berne J, Demetriades D et al. One hundred five cardiac injuries: a 2 year prospective evaluation. J Trauma, 1998, 44:1073-82.

22. Hoyt DB, Bulger EM, Knudson MM et al. Death in the operating room: an analysis of a multi-center experience. J Trauma, 1994, 37: 426-32.

23. Saad Júnior R, Aguiar Neto JR, Dorgan Neto V et al. Trauma de tórax: análise de 114 doentes. Classificação, indicação operatória e mortalidade. J Pneumol, 1995, 21: 79-82.

24. Asensio J. Exsanguination from penetrating injuries. Trauma Q, 1990, 6:1-25.

25. Naughton MJ, Brissie RM, Bessey PQ et al. Demography of penetrating cardiac trauma. Ann Surg, 1989, 209: 676-81.

26. Coimbra R, Hoyt $\mathrm{D}$, Winchell $\mathrm{R}$ et al. The ongoing challege of retroperitoneal injuries. Am J Surg, 1996, 172: 541-4.

27. Ombrellaro MP, Freeman MB, Stevens SL et al. Predictors of survival after inferior vena cava injuries. Am Surg, 1997, 63: 178-83.

28. Davis JW, Kaups KL, Parks SN. Base deficit is superior to $\mathrm{pH}$ in evaluating clearance of acidosis after traumatic shock. J Trauma, 1998, 44: 114-8.

29. Cosgriff N, Moore EE, Sauauia A et al. Predicting life-threatening coagulopathy in the massively transfused trauma patient: hypothermia and acidoses revisited. J Trauma, 1997, 42: 857-61.

30. Sauaia A, Moore FA, Moore EE et al. Early predictors of postinjury multiple organ failure. Arch Surg, 1994, 129: 39-45.

31. Gofrit ON, Leibovici D, Shapira SC et al. The trimodal death distribution of trauma victims: military experience from the Lebanon War. Mil Med, 1997, 162 24-6.
32. Carrillo C, Fogler RJ, Shaftan GW. Delayed gastrointestinal reconstruction following massive abdominal trauma. J Trauma, 1993, 34: 233-5.

33. Feliciano D, Moore EE, Mattox KL. Damage control and alternative wound closures in abdominal trauma. In: Trauma. 3. Ed. Stamford, Conn., Appleton \& Lange, 1996. P. 717-31.

34. Garrison JR, Richardson JD, Hilakos AS et al. Predicting the need to pack early for severe intra-abdominal hemorrhage. J Trauma, 1996, 40: 923-7.

35. Rotondo MF, Zonies DH. The damage control sequence and underlying logic. Surg Clin North Am, 1997, 77: 76177.

36. Hirshberg A \& Walden R. Damage control for abdominal trauma. Surg Clin North Am, 1997, 77: 813-20.

38. Krishna G, Sleigh JW, Rahman H. Physiological predictors of death in exsanguinating trauma patients undergoing convencional trauma surgery. Aust N Z J Surg, 1998, 68: 826-9.

Endereço para correspondência:

Dr. José Gustavo Parreira

Rua Dona Veridiana, 167/83

01228-020 — São Paulo-SP 